\title{
Current management aspects in adult congenital heart disease: non-surgical closure of patent foramen ovale
}

\author{
Kaivan Vaidya $^{1,2}$, Chinmay Khandkar ${ }^{1,2}$, David Celermajer ${ }^{1,2}$ \\ ${ }^{1}$ Department of Cardiology, Royal Prince Alfred Hospital, Sydney, Australia; ${ }^{2}$ Central Clinical School, Faculty of Medicine, University of Sydney, \\ Sydney, Australia \\ Contributions: (I) Conception and design: All authors; (II) Administrative support: None; (III) Provision of study materials or patients: None; \\ (IV) Collection and assembly of data: All authors; (V) Data analysis and interpretation: All authors; (VI) Manuscript writing: All authors; (VII) Final \\ approval of manuscript: All authors. \\ Correspondence to: Kaivan Vaidya. Department of Cardiology, Royal Prince Alfred Hospital, Sydney, Australia. Email: kaivan.vaidya@gmail.com.
}

\begin{abstract}
A patent foramen ovale $(\mathrm{PFO})$ is a remnant interatrial communication, best diagnosed with transoesophageal echocardiography (TOE) and bubble study. Although quite common and often asymptomatic, PFO is associated with cryptogenic stroke and migraine. Approximately one-half of patients with a cryptogenic stroke have a $\mathrm{PFO}$, and the dilemma regarding whether or not to proceed with percutaneous device closure, to reduce the risk of future recurrent events due to paradoxical embolism, has been subject to debate for nearly two decades. Despite promising observational data, initial randomised clinical trials failed to demonstrate superiority of closure over medical therapy. However, long-term follow-up data from one of these early trials, combined with two new randomised trials, have provided more evidence for the benefits of closure in selected patients. This new evidence suggests that younger patients with high-risk features such as an atrial septal aneurysm (ASA) or large interatrial shunt are more likely to benefit from PFO closure, after fastidious exclusion of an alternative cause for the index stroke. However, issues which require further clarification include whether anticoagulant therapy is preferable to antiplatelet therapy for medical management, and which particular type of closure device is optimal. Finally, despite promising retrospective observational data suggesting improvement in migraine attacks after PFO closure, high quality evidence is lacking in this regard.
\end{abstract}

Keywords: Cryptogenic stroke; transient ischaemic attack (TIA); migraine; patent foramen ovale (PFO)

Submitted Jul 10, 2018. Accepted for publication Aug 16, 2018.

doi: $10.21037 / \mathrm{cdt} .2018 .09 .09$

View this article at: http://dx.doi.org/10.21037/cdt.2018.09.09

\section{Introduction}

The foramen ovale is a natural connection between the atria during foetal development. In utero, oxygenated blood enters the right heart via the umbilical vein and bypasses the pulmonary circuit via the foramen ovale to enter systemic circulation. This right-to-left shunt forms the basis of foetal circulation.

From the fourth week of pregnancy, the primordial single atrium in the foetus is divided into two chambers via the fusion of interatrial structures: the septum primum and septum secundum (1). The septum primum grows upwards from the endocardial cushion (2). As it grows, perforations form due to pre-programmed apoptosis, which eventually fuse to form the foramen secundum. The septum secundum grows from the ventrocranial atrial wall down towards the $\mathrm{AV}$ valves, to incompletely overlap the septum primum. A small oval partition window remains, which is known as the foramen ovale (1). The residual septum primum forms the valve of the foramen ovale. This valve fuses with the septum secundum over several months after birth to usually obliterate this connection between the left and right heart. Failure to fuse completely results in an open communication between the left and right atria, known 


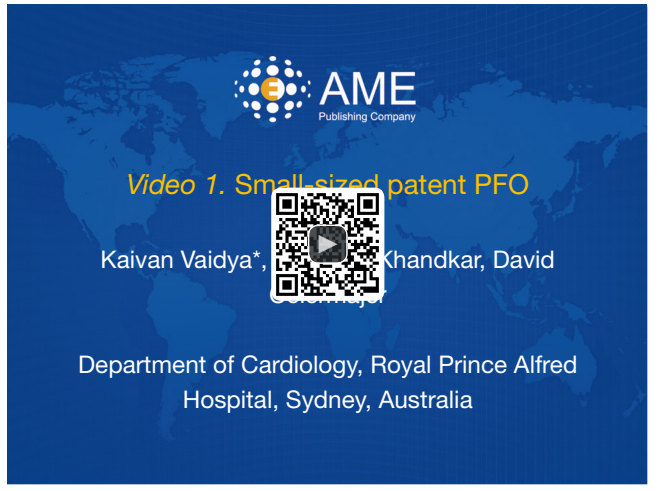

Figure 1 Small-sized patent PFO (9). PFO, patent foramen ovale. Available online: http://www.asvide.com/article/view/29132

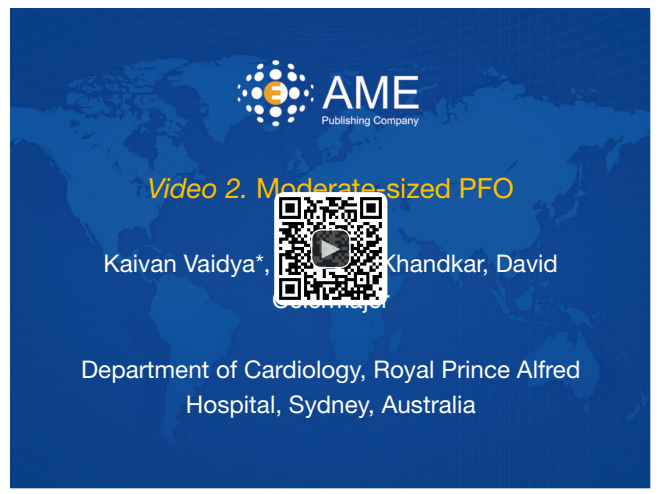

Figure 2 Moderate-sized PFO (10). PFO, patent foramen ovale. Available online: http://www.asvide.com/article/view/29134

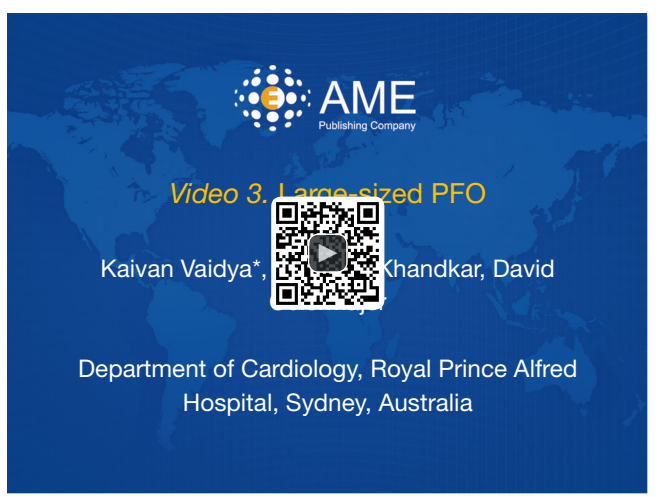

Figure 3 Large-sized PFO (11). PFO, patent foramen ovale. Available online: http://www.asvide.com/article/view/29135 as a patent foramen ovale (PFO). As long as this channel remains patent as an anatomical interatrial communication, shunting of blood may occur between the atria. A PFO is a common finding in the general adult population, with a prevalence of approximately 25\% (3). The reasons behind failure of PFO closure after birth are not well understood, and are likely due to multifactorial inheritance (4).

\section{Diagnosis}

The most accurate diagnostic method for PFOs in vivo is by right-heart catheterisation, where the catheter crosses the interatrial septum into the left atrium. However, with great improvements in modern echocardiography, angiography is now rarely required (2). A PFO can be detected with non-invasive imaging by transoesophageal echocardiography (TOE) or transthoracic echocardiography (TTE); and inferred from transcranial Doppler (TCD) (1).

TOE with bubble study is currently the accepted standard for non-invasive diagnosis of $\mathrm{PFO}$, with a mean sensitivity of $89.0 \%$ and a specificity of $91.2 \%$ (5). In order to diagnose a $\mathrm{PFO}$, the left atrium is visualised whilst a peripheral vein is injected with agitated saline. A diagnosis of PFO is valid if any micro-bubbles are visualised in the left atrium within three cardiac cycles from maximal right atrial opacification (6). The PFO can also be visualised on TOE, as a flap opening in the muscular wall of the septum secundum (7). The study is usually conducted with a provocation manoeuvre such as the Valsalva manoeuvre, in order to raise intra-thoracic pressures to subsequently increase the right-to-left shunt. This was shown to increase sensitivity, but may be suboptimal in TOE due to sedation (8). The biggest advantage of TOE compared to other non-invasive imaging modalities is that it provides visualisation of the right-to-left shunt to differentiate between a PFO and an atrial septal defect (ASD) or intrapulmonary shunt. It also quantifies shunt size, and allows operators to document anatomical PFO characteristics for potential closure device planning. Figures 1-3 demonstrates an example each of a small, moderate, and large-sized PFO. Furthermore, TOE allows optimal visualisation of other anatomical structures known to be associated with a PFO. These include a prominent Eustachian valve (directs blood 
from the inferior vena cava to the foramen ovale), Chiari network (fenestrated mobile network of filamentous fibres in the right atrium), as well as an atrial septal aneurysm (ASA), which is defined as redundant and hypermobile interatrial septum primum in the region of the fossa ovalis with phasic excursion of at least $10 \mathrm{~mm}$ during the cardiorespiratory cycle.

TTE can also be used to diagnose a PFO, however with less sensitivity in comparison to TOE $(6,12,13)$. The use of harmonic imaging and colour Doppler with TTE may increase the sensitivity of detection from $46 \%$ to $90 \%(5,7)$. However, TTE may be preferred in cases where a transoesophageal probe poses high risk, such as in patients with oesophageal varices or strictures. One study showed promising sensitivity and specificity for PFO detection using TTE with Doppler flow imaging across the mitral valve, i.e., transmitral Doppler (TMD), to detect the passage of bubbles from the left atrium to left ventricle (14). Further trials are required to validate this technique. The American Society of Echocardiography guidelines recommend using TTE as a screening tool, with escalation to TOE if the shunt cannot be demonstrated with colour Doppler or injection of aggravated saline (15).

TCD involves placing an ultrasound probe at the temple to visualise any of the anterior, middle, or posterior cerebral arteries. Agitated saline contrast is injected, and the arteries are examined for any presence of micro-bubbles to confirm a right-to-left shunt. A 2014 meta-analysis showed that TCD with colour Doppler (c-TCD) had comparable sensitivity (97\%) and specificity (93\%) to the gold standard of TOE in detecting a right-to-left shunt (16). The biggest disadvantage of TCD is the lack of visualisation of the shunt, thus lacking the ability to differentiate between PFO and other rightto-left shunts. Given the ease of use, accuracy, and high sensitivity of this imaging modality, there may be a possible role for c-TCD as a screening tool preceding TTE (7).

The above imaging modalities rely on the principle of detecting agitated saline contrast in either the left heart or systemic circulation. The antecubital fossa is most commonly used as the site of injection due to ease of access. The alternative femoral approach is perceived to have greater risk, with a higher risk of complications such as false aneurysm or arteriovenous fistula formation (17). However, contrast injection via the femoral vein is associated with significantly higher detection rates for $\mathrm{PFO}(18,19)$. The prevailing hypothesis behind this difference is that of caval inflow patterns, with the inferior vena cava directing blood flow to the interatrial septum, unlike the super vena cava which streams blood to the body of the atrium (20).

\section{PFO closure}

PFOs are mostly asymptomatic, but have been shown to be associated several clinical syndromes including cryptogenic stroke, migraine headache, decompression sickness and air embolism in divers, and the platypnoea-orthodeoxia syndrome (21). Thus, percutaneous PFO closure has naturally been an attractive therapeutic intervention, especially with modern advances in interventional catheter technology.

\section{Cryptogenic stroke}

Cryptogenic stroke refers to ischaemic cerebral events with no apparent cause despite extensive diagnostic evaluation, including exclusion of large-artery atherosclerotic disease, cardioembolic sources, small-vessel occlusive disease (lacunar stroke), hypercoagulable disorders, and arterial dissection. Approximately $25-40 \%$ of all ischaemic strokes or transient ischaemic attacks (TIA) in patients under 60 years of age are classed as cryptogenic $(22,23)$. Studies suggest a causal relationship between PFO and cryptogenic stroke, reporting an increased prevalence of PFO (up to 60\%) in young adults with strokes of otherwise unidentifiable cause (24-26). Several case-control studies have shown that PFO prevalence was three times higher in patients with cryptogenic stroke than in controls without stroke or stroke patients with a known cause, and this difference was amplified to five times higher in younger adults (under 55 years of age) with cryptogenic stroke. The association between $\mathrm{PFO}$ and cryptogenic stroke is stronger if there is an ASA and/or substantial or spontaneous right-to-left shunt $(5,23,27,28)$. An ASA associated with a $\mathrm{PFO}$ increases the risk of initial [odds ratio (OR) 4.96] and recurrent stroke (OR 23.9), as it can open the PFO with the cardiac cycle (5).

The pathophysiology of stroke in patients with a PFO may be multifactorial. The prevailing hypothesis currently is that of a paradoxical embolism, i.e., a PFO allows blood-borne material (usually venous thrombus) to bypass the pulmonary circuit to enter systemic arterial circulation, and subsequently pass into the cerebral vasculature (29). This is supported by case-reports documenting visualised thrombus in the interatrial septum in the setting of embolic stroke $(30,31)$. However, alternative hypotheses also speculate formation of thrombotic material in situ within the PFO (at the level 
Table 1 The RoPE score for evaluating whether a cryptogenic stroke is PFO-attributable

\begin{tabular}{lc}
\hline Characteristic & Points \\
\hline Patient characteristic & +1 \\
No history of hypertension & +1 \\
No history of diabetes & +1 \\
No history of stroke or TIA & +1 \\
Non-smoker & +1 \\
Cortical infarct on imaging & \\
Age (years) & +5 \\
18-29 & +4 \\
$30-39$ & +3 \\
$40-49$ & +2 \\
$50-59$ & +1 \\
$60-69$ & +0 \\
$\geq 70$ & $0-10$ \\
Total score & \\
\hline
\end{tabular}

PFO, patent foramen ovale.

of the foramen channel or ASA), with the potential for embolization $(23,27)$.

Given the consideration of percutaneous closure of PFO in patients with cryptogenic strokes, it is naturally important to establish when such events are in fact PFO-attributable (as opposed to an alternate cause with an incidental PFO finding) and which cryptogenic stroke patients with $\mathrm{PFO}$ are at a high risk of recurrence. Patients with a high "attributable recurrence risk" would benefit most from PFO closure for secondary stroke prevention. A pooled database of 3,023 patients with cryptogenic strokes sought to determine this $(32,33)$ with a 10 -point risk stratification system named the Risk of Paradoxical Embolism (RoPE) score (Table 1), incorporating age, presence of cortical infarct on imaging, and vascular risk factors (hypertension, diabetes, prior stroke/TIA, smoking). PFO prevalence increased from $23 \%$ in the lowest RoPE stratum (older patients with risk factors and non-cortical events) to $73 \%$ in the highest RoPE stratum (younger patients, superficial cortical infarcts, less risk factors). The lowest stratum conferred a $20 \% 2$-year stroke/TIA recurrence rate with $0 \%$ of index events attributable to $\mathrm{PFO}$, whilst the highest stratum conferred a $2 \% 2$-year recurrence rate with $88 \%$ of index events attributable to PFO. This study demonstrated that recurrence risk was substantially lower in patients most likely to have a PFO-attributable stroke, which underscores the challenges of patient selection for catheter-based PFO closure. It did, however, lay the groundwork for further studies seeking to identify variables capable of predicting higher recurrence rates among those with higher RoPE scores, particularly echocardiographic features such as spontaneous shunting at rest, shunt size, and an associated ASA (34).

Conventional therapy for cryptogenic strokes revolves around medical management. However, there has been a recent growing interest in transcatheter closure of PFOs, with multiple randomised control trials (RCTs) conducted head-to-head with medical therapy to evaluate stroke recurrence rates. An early meta-analysis (27) of over 2,000 patients showed that the adjusted annual recurrence rate of stroke/TIA was $1.86 \%$ in the closure arm compared to $5.42 \%$ in the medical treatment arm $[\mathrm{P}<0.001$; number needed to treat $(\mathrm{NNT})=28]$, and the adjusted rate of stroke/ TIA at 1 year was $2.71 \%$ and $7.07 \%$ in the closure and medical therapy arms respectively $(\mathrm{P}<0.001 ; \mathrm{NNT}=23)$. Subsequently, however, there have been several large RCTs seeking to establish the superiority of device closure over medical therapy for the prevention of recurrent strokes, with varying results. Three initial RCTs did not demonstrate any significant benefit of closure, but two RCTs have more recently been published, as well as long term results from a previous initial RCT, which have substantially changed the landscape of transcatheter PFO closure for secondary prevention of recurrent cerebrovascular events.

\section{The first set of randomized trials from 2012 to 2016}

CLOSURE-1 was the first multicentre RCT comparing percutaneous closure with medical therapy over two years follow-up, in 909 patients aged 18 to 60 with a cryptogenic stroke and PFO (35) (Table 2). Percutaneous closure was with the STARFlex device (NMT Medical) and after the procedure, patients were given aspirin and clopidogrel for 6 months and aspirin alone up to 2 years. Patients assigned to medical therapy were treated with warfarin [target international normalised ratio (INR) 2.0 to 3.0], aspirin, or both. Device implantation was successful in $89.4 \%$ of patients, and at 2 years there was no significant difference in recurrent stroke/TIA between the device $(5.5 \%)$ and medical therapy (6.8\%) groups [adjusted hazard ratio (HR) $0.78,95 \%$ confidence intervals $(\mathrm{CI}) 0.45-1.35, \mathrm{P}=0.37$ ). The respective rates in the device and medical arms were $2.9 \%$ and $3.1 \%$ for stroke $(\mathrm{P}=0.79)$ and $3.1 \%$ and $4.1 \%$ for TIA $(\mathrm{P}=0.44)$. There was also no difference in treatment 
Table 2 Summary of clinical trials comparing percutaneous PFO closure to medical therapy in cryptogenic stroke patients

\begin{tabular}{|c|c|c|c|c|c|}
\hline Study & $\begin{array}{l}\text { Patient cohort } \\
\text { (no. of patients) }\end{array}$ & Closure arm & Medical arm & Primary outcome & Results \\
\hline CLOSURE-1 & $\begin{array}{l}\text { Age } 18-60 \\
(\mathrm{n}=909)\end{array}$ & $\begin{array}{l}\text { STARFlex device, aspirin and } \\
\text { clopidogrel for } 6 \text { months, } \\
\text { aspirin alone up to } 2 \text { years }\end{array}$ & $\begin{array}{l}\text { Warfarin, aspirin, } \\
\text { or both }\end{array}$ & $\begin{array}{l}\text { Recurrence of } \\
\text { stroke, TIA, early } \\
\text { all-cause mortality, } \\
\text { late neurological } \\
\text { mortality }\end{array}$ & $\begin{array}{l}\text { No difference } \\
\text { between groups } \\
\text { (adjusted HR } \\
0.78, P=0.37 \text { ) }\end{array}$ \\
\hline $\begin{array}{l}\text { RESPECT } \\
\text { (extended) }\end{array}$ & $\begin{array}{l}\text { Age } 18-60 \\
(\mathrm{n}=980)\end{array}$ & $\begin{array}{l}\text { Amplatzer PFO Occluder, } \\
\text { aspirin and clopidogrel for } \\
1 \text { month, then aspirin alone } \\
\text { for further } 5 \text { months }\end{array}$ & $\begin{array}{l}\text { Aspirin, clopidogrel, } \\
\text { warfarin, aspirin with } \\
\text { extended-release } \\
\text { dipyridamole, or aspirin } \\
\text { and clopidogrel }\end{array}$ & $\begin{array}{l}\text { Recurrent } \\
\text { ischaemic stroke } \\
\text { or early death }\end{array}$ & $\begin{array}{l}\text { Lower ischaemic } \\
\text { stroke in the } \\
\text { device arm (HR } \\
0.50, P=0.02) \text { and } \\
\text { no early mortality } \\
\text { in either group }\end{array}$ \\
\hline CLOSE & $\begin{array}{l}\text { Age 16-60. PFO } \\
\text { associated with } \\
\text { ASA or large shunt } \\
\quad(n=663)\end{array}$ & $\begin{array}{l}\text { One of } 11 \text { closure devices } \\
\text { (commonest Amplatzer } \\
\text { PFO Occluder), aspirin and } \\
\text { clopidogrel for } 3 \text { months, then } \\
\text { single agent alone }\end{array}$ & $\begin{array}{l}\text { Aspirin, clopidogrel, aspirin } \\
\text { plus clopidogrel, aspirin } \\
\text { with extended-release } \\
\text { dipyridamole, warfarin, or } \\
\text { direct oral anticoagulant }\end{array}$ & Stroke & $\begin{array}{l}\text { Recurrent stroke } \\
\text { occurred in the } \\
\text { medication arm } \\
\text { only (HR 0.03, } \\
\mathrm{P}<0.001 \text { ) }\end{array}$ \\
\hline Gore REDUCE & $\begin{array}{l}\text { Age } 18-59 \\
(\mathrm{n}=664)\end{array}$ & $\begin{array}{l}\text { Gore Helex/Cardioform Septal } \\
\text { Occluder Clopidogrel loading } \\
\text { dose then } 3 \text { days of once daily } \\
\text { therapy, then aspirin alone, } \\
\text { clopidogrel alone, or aspirin } \\
\text { with dipyridamole alone }\end{array}$ & $\begin{array}{l}\text { Aspirin, clopidogrel, or } \\
\text { aspirin with dipyridamole }\end{array}$ & $\begin{array}{l}\text { Clinical ischaemic } \\
\text { stroke, or incidence } \\
\text { of new brain } \\
\text { infarction on MRI }\end{array}$ & $\begin{array}{l}\text { Lower incidence } \\
\text { of recurrent } \\
\text { clinical ischaemic } \\
\text { stroke (HR } 0.23 \text {, } \\
\mathrm{P}=0.002) \text { and new } \\
\text { brain infarction } \\
\text { (RR } 0.51, \mathrm{P}=0.04) \\
\text { in the closure arm }\end{array}$ \\
\hline
\end{tabular}

PFO, patent foramen ovale; TIA, transient ischaemic attack; ASA, atrial septal aneurysm.

effect between various subgroups, including those defined by shunt size or the presence/absence of an ASA. No deaths occurred during the study, and there were no significant differences in the rates of serious adverse events between the two groups $(\mathrm{P}=0.90)$. However, the closure group had a 3.2\% rate of major vascular complications and substantially higher rates of atrial fibrillation (AF) $(5.7 \%$ vs. $0.7 \%, \mathrm{P}<0.001)$; most of this $(61 \%)$ was periprocedural, within 30 days of implantation. A purported explanation for the negative result in this trial is issues with the STARFlex septal closure device itself, as the rate of effective closure at 6 months was $86.1 \%$ (14\% had residual right-to-left shunting), although none of the patients in the closure group who had a recurrent stroke or TIA had a residual shunt.

The PC trial was a multicentre RCT comparing closure with medical therapy in 414 patients less than 60 years of age with PFO and cryptogenic ischaemic stroke, TIA or an extracranial peripheral thromboembolic event (36) (Table 2). The closure device used was the Amplatzer PFO Occluder (St. Jude Medical) and antithrombotic treatment 
post-procedure consisted of aspirin for at least 5 to 6 months and ticlopidine or clopidogrel for 1 to 6 months. In the medical therapy arm, treatment was antiplatelet and/or anticoagulation. The primary prespecified endpoint was a composite of death, nonfatal stroke, TIA, or peripheral embolism; with a mean duration of follow-up of 4.1 and 4.0 years in the closure and medical therapy arms respectively. Implantation was deemed to be successful in 188 of $196(95.9 \%)$ in the closure group, and at 6 months TOE showed effective closure with no/minimal shunt in $95.9 \%$ of these. In the medical therapy arm, antiplatelet therapy was more commonly administered $(73.8 \%)$ than oral anticoagulation (30.5\%). There was no difference in the primary outcome between the two groups $(3.4 \%$ vs. $5.2 \%$ in device $v s$. medical arms respectively) (HR 0.63, 95\% CI: $0.24-1.62, \mathrm{P}=0.34)$ over the study period. Stroke $(0.5 \%$ vs. $2.4 \%$, HR $0.20, \mathrm{P}=0.14)$ and TIA $(2.5 \%$ vs. $3.3 \%$, HR $0.71, \mathrm{P}=0.56$ ) both occurred less in the closure group but neither demonstrated a statistically significant difference. There was no interaction detected between the two arms with subgroup analyses either, including with age $(\mathrm{P}=0.10)$ and presence of ASA $(\mathrm{P}=0.09)$. In the closure and medical treatment groups respectively, serious adverse events occurred in $21.1 \%$ and $17.6 \%$ of patients, new onset AF was detected in $2.9 \%$ and $1.0 \%$ (HR 3.15, P=0.16), and bleeding occurred in $3.9 \%$ and $5.7 \%$ (HR $0.66, \mathrm{P}=0.40$ ). The major limitation of the PC trial was that it was "underpowered" and hence prone to an inability to detect a clinically relevant benefit of PFO closure. Furthermore, inclusion of patients with a noncerebral peripheral thromboembolic event led to recruitment of a study population different to most other PFO studies, and there was a crossover rate of $13.3 \%$ of patients from the medical therapy arm who subsequently underwent PFO closure.

Finally, the RESPECT trial was a prospective, multicentre RCT comparing closure with medical therapy in 980 patients aged 18 to 60 (mean age 45.9 years) with a cryptogenic stroke and PFO identified (37) (Table 2). In this study, shunt size was classified as grade 1 (1 to 9 microbubbles within 3 cardiac cycles), grade 2 (10 to 20), and grade 3 (over 20). The closure device used was the Amplatzer PFO Occluder (followed by aspirin and clopidogrel for one month, then aspirin alone for 5 months), and the medical therapy group received either aspirin (46.5\%), clopidogrel (14.0\%), warfarin (25.2\%), aspirin with extended-release dipyridamole $(8.1 \%)$, or aspirin and clopidogrel (6.2\%), at physician discretion. The primary efficacy endpoint was a composite of recurrent ischaemic stroke or early death, with a mean follow-up of 2.6 years, although there was a substantially higher dropout rate in the medical $(17.2 \%)$ than closure $(9.2 \%)$ arm. In the closure group, the rate of procedural success with no in-hospital serious adverse events was $96.1 \%$. Again, although there was a signal towards less events in the closure group (1.8\%) than medical treatment (3.3\%) (HR 0.49, 95\% CI: 0.22-1.11, $\mathrm{P}=0.08$ ), this was not statistically significant. However, the difference between the two groups was significant in the prespecified per-protocol cohort (HR 0.37, $\mathrm{P}=0.03$ ) and in the as-treated cohort ( $\mathrm{HR} 0.27, \mathrm{P}=0.007)$, both favouring closure over medical therapy. Subgroup analyses suggested that closure may have a greater benefit in patients with a substantial right-to-left shunt $(\mathrm{P}=0.01)$ and in those with an ASA $(\mathrm{P}=0.02)$. The rate of serious adverse events did not differ significantly between the closure $(23.0 \%)$ and medical therapy $(21.6 \%)$ groups. The total incidence of AF did not differ between the closure (3.0\%) and medical (1.5\%) arms $(\mathrm{P}=0.13)$. Importantly, in the closure group, no recurrent strokes from AF or device thrombosis occurred. The key issue with the RESPECT trial was the lack of an adequate follow-up period, given the low number of events registered in younger patients who are at risk over an extended period. Furthermore, the difference in dropout rates between the two groups, which resulted in an unequal duration of exposure to the risk of recurrence, complicates result interpretation.

These three trials did not meet their expected outcome as they were quite limited by low numbers of recruited patients, lower-than-expected event rates, and short follow-up periods (a postulated benefit needed more time to become significant). After the publication of these trials, several meta-analyses were conducted to increase the sample size and evaluate whether closure was clinically superior to medical therapy. One such systematic review (38) of the three trials showed that across 2,303 patients, device closure was more effective than medical therapy for prevention of recurrent neurological events (HR 0.67, 95\% CI: 0.44-1.0, $\mathrm{P}=0.05)$, with even more robust results when only $\mathrm{PC}$ and RESPECT were pooled (HR 0.54, 95\% CI: 0.29-1.01), both of which used the Amplatzer PFO Occluder. These findings were further supported by a pooled individualpatient meta-analysis [relative risk (RR) 0.39, 95\% CI: 0.17-0.84) (39) and a study-level network meta-analysis (HR 0.68, P=0.049) (40). The Amplatzer PFO Occluder in RESPECT and PC had higher effective closure rates than the STARFlex device in CLOSURE-1, without provoking more AF or device thrombosis which could 
result in a recurrent stroke (35-37). These results, as well as U.S. Food and Drug Administration (FDA) approval of the Amplatzer PFO Occluder in 2016, spurred further evaluation of whether percutaneous closure was in fact superior to medical therapy alone, in preventing recurrent cerebrovascular events.

\section{The second set of randomized trials and the current impression (2017 onwards)}

The CLOSE trial (41) was a multicentre RCT comparing transcatheter PFO closure, antiplatelet therapy alone, and oral anticoagulation therapy, in 663 patients aged 16 to 60 with a cryptogenic ischaemic stroke and PFO with an associated ASA or large right-to-left shunt (>30 microbubbles within three cardiac cycles), over a mean follow-up period of 5.3 years (Table 2). Alternative causes of stroke were excluded with a comprehensive list of investigations, but long-term electrocardiographic (ECG) monitoring to exclude occult $\mathrm{AF}$ was not performed. $\mathrm{PFO}$ closure was performed using one of 11 available implantable devices, most commonly the Amplatzer PFO Occluder $(121 / 235 ; 51.5 \%)$ or the Intrasept PFO Occluder (13.2\%). The closure group received aspirin and clopidogrel for 3 months then single agent alone; the antiplatelet therapy group received aspirin (86.7\%), clopidogrel (10.8\%), aspirin plus clopidogrel $(1.3 \%)$, or aspirin with extended-release dipyridamole $(1.3 \%)$; and the anticoagulation group received warfarin (93\%) with target INR 2.0 to 3.0 or direct oral anticoagulants (7\%). The primary efficacy outcome was stroke, and safety outcomes were major or fatal procedural or haemorrhagic complications. Successful PFO closure with no periprocedural complication and no/minimal residual shunt occurred in $88.6 \%$ of patients in the closure arm. At follow-up, no patients in the closure group had a stroke, compared to $14 / 235(6.0 \%)$ in the antiplatelet-only group (HR 0.03, 95\% CI: $0-0.26, \mathrm{P}<0.001$ ). Of these 14 , no explanation other than a PFO was found for the recurrent event, 9 had an ASA, and 5 had a large shunt but no aneurysm. The secondary composite outcome of stroke, TIA, or systemic embolism also occurred in fewer patients in the closure group than in the antiplatelet group $(3.4 \%$ vs. $8.9 \%$, HR 0.39, 95\% CI: 0.16-0.82, $\mathrm{P}=0.01)$. Follow-up echocardiography (mean 10.8 months) demonstrated that $93 \%$ of closure patients had no or minimal residual shunt. Serious adverse events occurred similarly across the closure $(35.7 \%)$ and anti-platelet $(33.2 \%)$ groups. Major procedural complications occurred in $5.9 \%$ of patients in the closure group, and in particular the rate of new-onset
AF or flutter was higher $(\mathrm{P}=0.02)$ in the closure group $[11 / 238(4.6 \%)]$ compared to the antiplatelet group [2/235 $(0.9 \%)]$. However, in the closure group, 10 out of $11 \mathrm{AF}$ cases occurred within one month of the procedure, and AF did not recur during a median follow-up period of 4.4 years.

In CLOSE, comparing antiplatelet and anticoagulation therapy, there were lower rates of stroke in the anticoagulation group with Kaplan-Meier 5-year estimates $1.5 \%$ vs. $3.8 \%$ in the antiplatelet cohort, however this analysis was underpowered to assess significance. In this study, results clearly showed that percutaneous PFO closure was associated with fewer recurrent strokes in patients with cryptogenic strokes and echocardiographic features such as presence of a large shunt or ASA, with an NNT $=20$ to prevent one stroke every 5 years. The restricted patient characteristics may explain the positive findings compared to older trials, as only patients with "high-risk" features associated with cryptogenic stroke were enrolled. The risk of stroke from AF induced by $\mathrm{PFO}$ closure remains to be determined, but may not be clinically significant given the lack of recurrence of AF in this study with over 4 years of follow-up. Although there was no prolonged ECG monitoring to exclude occult AF, the yield of this is low in younger patients and does not explain the lower rates of stroke recurrence in the closure group.

Extended results from the RESPECT trial were also published in 2017 (42), with a longer follow-up period of the same 980 patients, now over median 5.9 years (previously 2.1 years) (Table 2). The dropout rate was 33.3\% in the medical therapy and $20.8 \%$ in the PFO closure groups, resulting in a significant between-group difference in median duration of follow-up $(\mathrm{P}<0.001)$. Recurrent ischaemic stroke occurred in 18 patients $(3.6 \%)$ in the Amplatzer PFO Occluder closure group and in 28 patients $(5.8 \%)$ in the medical therapy group (HR $0.55,95 \%$ CI: $0.31-1.00, \mathrm{P}=0.046$ ), and no early deaths occurred in either group. This interaction remained significant with multiple imputation analysis and covariate adjustment (HR 0.50, $\mathrm{P}=0.02$ ). In addition, this extended follow-up demonstrated a highly significant $62 \%$ relative risk reduction for recurrent stroke of unknown aetiology, in favour of closure. When assessing secondary endpoints, there were substantially fewer recurrent cryptogenic ischaemic strokes in the closure $v s$. the medical therapy cohorts (HR $0.08, \mathrm{P}=0.01$ ) but there was no significant difference in incidence of TIA (HR 0.64, $\mathrm{P}=0.16)$. Moreover, subgroup analyses for treatment effect heterogeneity suggested that the benefit of PFO closure may be greatest amongst patients with an ASA (HR 0.20, 
$\mathrm{P}=0.005$ ), a substantial (grade 3) shunt (HR 0.26, $\mathrm{P}=0.005$ ), and patients whose planned medical regimen (if assigned to the control group) included antiplatelets rather than anticoagulants (HR 0.38, $\mathrm{P}=0.007$ ). The overall rate of serious adverse events was $40.3 \%$ in the $\mathrm{PFO}$ closure group and $36.0 \%$ in the medical therapy group $(\mathrm{P}=0.17)$, with higher rates of pulmonary emboli (HR 3.48, $\mathrm{P}=0.04)$ and deep venous thrombosis (DVT) (HR 4.44, $\mathrm{P}=0.14$ ) in the closure group. There were 25 serious device or procedurerelated adverse events including ischaemic stroke $(0.4 \%)$, residual shunt requiring closure $(0.4 \%)$, cardiac perforation, and cardiac thrombus. There were seven periprocedural episodes of AF which occurred in the closure group which all resolved prior to patient discharge, and after the periprocedural period there was no difference in $\mathrm{AF}$ rates between the two groups (HR 1.47, $\mathrm{P}=0.36$ ). This extended follow-up of the initial RESPECT trial showed that PFO closure in cryptogenic stroke was associated with a lower rate of recurrent ischaemic strokes than medical therapy, with an NNT $=42$ to prevent one stroke over 5 years. The higher rates of venous thromboemboli in the closure group likely reflected the lower rates of anticoagulation in this group (the medical therapy group had patients on warfarin). Again, there was no prolonged ECG monitoring to detect occult $\mathrm{AF}$, and the higher dropout rate in the medical arm compared to the closure arm may have resulted in an unequal duration of exposure to the risk of recurrent ischaemic strokes.

The Gore REDUCE trial (43) was a multicentre RCT comparing $\mathrm{PFO}$ closure with antiplatelet therapy alone (2:1 ratio) in 664 patients aged 18 to 59 years (mean age 45.2), with a cryptogenic ischaemic stroke and PFO with right-to-left shunt, over a median 3.2 year follow-up duration (Table 2). Shunt size was defined as small (1 to 5 microbubbles within 3 cardiac cycles), moderate (6 to 25), or large (over 25); $81 \%$ of PFOs were moderate or large in this study. The index stroke was defined as cryptogenic after exclusion of all other possible mechanisms, although prolonged ECG monitoring was not required. PFO closure was performed with either the Gore Helex Septal Occluder (HELEX) $(\mathrm{n}=158)$ or the Gore Cardioform Septal Occluder $(\mathrm{GSO})(\mathrm{n}=250)$. Antiplatelet therapy included aspirin, clopidogrel, or aspirin plus dipyridamole; and the closure group received a clopidogrel loading dose then 3 days of once daily therapy, followed by any of the aforementioned antiplatelet regimens. The primary endpoints were freedom from a clinical ischaemic stroke over at least 24 months, and incidence of new brain infarction (clinical or silent) detected between the baseline and 24-month brain imaging [usually magnetic resonance imaging (MRI)]. Premature discontinuation from the trial occurred in $8.8 \%$ of the closure group and $14.8 \%$ of the antiplatelet group. Complete PFO closure with a study device was achieved in $73.2 \%$ immediately post-procedure and in $75.6 \%$ at 12 months on echocardiography, while effective closure (freedom from large shunt) was achieved in $94.5 \%$ at 12 months. In the antiplatelet arm, 14 patients (6.3\%) underwent subsequent closure. Transcatheter closure was superior to antiplatelet therapy for preventing both recurrent clinical ischaemic stroke (1.4\% vs. 5.4\%, HR 0.23 , 95\% CI: 0.09-0.62, P=0.002), and new brain infarction (clinical or silent) on imaging $(5.7 \%$ vs. $11.3 \%$, RR 0.51 , 95\% CI: 0.29-0.91, $\mathrm{P}=0.04)$. There was no significant between-group difference in the rate of silent ischaemic events alone. Serious adverse events occurred in $23.1 \%$ and $27.8 \%$ of the closure and antiplatelet cohorts respectively $(\mathrm{P}=0.22)$, with two non-procedural deaths in the closure arm and none in the medical arm. Procedure-related serious adverse events occurred in $2.5 \%$ of closure patients, and device-related serious adverse events in $1.4 \%$, including device dislocation, device-related thrombosis, and aortic dissection. There was no difference between groups in the risk of bleeding $(\mathrm{P}=0.57)$, and atrial fibrillation or flutter occurred in more patients in the closure $(6.6 \%)$ group than antiplatelet $(0.4 \%)$ group $(\mathrm{P}<0.001)$. However, $83 \%$ of these $\mathrm{AF} /$ flutter cases were within 45 days of the procedure, $59 \%$ resolved within 2 weeks of onset, and only one had a recurrent stroke.

Results from the Gore REDUCE trial therefore supported the findings of other trials that transcatheter PFO closure was superior to medical therapy alone in patients with cryptogenic stroke, with an NNT $=28$ to prevent one stroke in 24 months. Importantly, this trial carefully selected patients who were more likely to have strokes attributable to PFO (e.g., excluding smaller lacunar strokes and uncontrolled vascular risk factors), hence increasing the likelihood that PFO closure would be effective.

Finally, the most recent trial published confirming these findings is the smaller DEFENSE-PFO trial, a multicentre RCT of 120 patients (mean age 51.8 years) with cryptogenic stroke and high-risk PFO who were randomised to either transcatheter closure or medical therapy, with a median duration of follow-up of 2.8 years (Table 2). This trial sought to evaluate whether benefits of PFO closure can be determined based on morphological characteristics, to select optimal candidates for the procedure. High-risk 
PFOs included those with an ASA, hypermobility (phasic septal excursion at least $10 \mathrm{~mm}$ ), or PFO size (maximum separation of the septum primum from the secundum of at least $2 \mathrm{~mm}$ ). The primary endpoint was a composite of stroke, vascular death, or major bleeding during 2 years of follow-up; and closure was performed with the Amplatzer PFO Occluder (all closures were successful). In the closure group, dual antiplatelet therapy (aspirin and clopidogrel) was recommended for at least 6 months, and in the medical therapy group patients received aspirin, aspirin plus clopidogrel, aspirin plus cilostazol, or warfarin (INR target 2.0 to 3.0). The primary endpoint occurred in 6 of 60 patients in the medication group only (2-year event rate $12.9 \%, 2$-year ischaemic stroke rate $10.5 \%, \mathrm{P}=0.023$ ). Major procedural complications in the closure group included development of AF $(\mathrm{n}=2)$, pericardial effusion $(\mathrm{n}=1)$, and femoral pseudoaneurysm $(\mathrm{n}=1)$. In this study, the rate of the primary endpoint was substantially lower with closure of high-risk PFOs in a select group of patients with cryptogenic stroke, compared to medical therapy alone, with NNT $=10$ to avoid one stroke in 2 years. The DEFENSE-PFO trial was similar to the CLOSE trial, in that both included stringent entry criteria regarding the anatomical characteristics of the atrial septum and PFO. Interestingly, both the CLOSE trial and DEFENSE-PFO showed no occurrence of stroke in patients who underwent PFO closure, suggesting that perhaps the beneficial effect of closure can be maximised in this select high-risk group.

A systematic review and study-level meta-analysis of all six RCTs $(n=3,560)$ thus far confirmed that PFO closure was superior to medical therapy for prevention of recurrent stroke (RR 0.36, 95\% CI: 0.17-0.79, $\mathrm{P}=0.01$ ), with an annual incidence of $1.27 \%$ in the medical therapy group (44). The effect of PFO closure on stroke recurrence was greatest in patients with an ASA or large shunt (RR 0.27, 95\% CI: $0.11-0.70, \mathrm{P}=0.01)$. There was no difference between groups in the incidence of TIAs (RR 0.85, $\mathrm{P}=0.38$ ) or major bleeding (RR 0.97, $\mathrm{P}=0.94$ ). New-onset AF was more significantly more frequent in patients randomised to device closure $v s$. antithrombotic therapy (RR 4.33, 95\% CI: 2.37-7.89, $\mathrm{P}<0.001$ ).

The discrepancy in statistical significance between the more recent trials and previous results is likely multifactorial. Stricter selection and exclusion criteria in the newer trials selected out patients with cryptogenic stroke more likely to be attributable to a PFO, and also honed in on potential "high-risk" features that predispose most to recurrent paradoxical cerebrovascular embolic events. Other contributing factors include the longer period of follow-up (due to the low event rate of recurrent stroke), and the fact that during the earlier trials, off-label PFO closure was often performed outside the trial setting which introduced selection and retention biases by potentially excluding high-risk patients, and diluting the actual beneficial effect of closure when high-risk patients randomised to medical therapy had off-label closure performed $(5,23,33,38,44,45)$.

Although the aforementioned trials and meta-analysis clearly delineate the fact that the annual stroke risk is overall low $(1-2 \%)$ and hence the absolute reduction in events with PFO closure is modest, cumulative lifetime benefit is probably meaningful for young patients who are undergoing a relatively safe one-off procedure. The meta-analysis, as well as DEFENSE-PFO and CLOSE, suggest that patients most likely to benefit are clearly those aged 18 to 60 years and with certain anatomical features associated with a higher risk of stroke recurrence, i.e., an ASA or a large shunt. This is pathophysiologically plausible, as increased septal mobility and a larger interatrial channel both enhance the probability of paradoxical embolus by mechanically directing more blood from the inferior vena cava to the PFO (44). The safety of device closure was comparable to medical therapy with respect to overall serious adverse events; although a higher rate of new-onset atrial fibrillation was clearly observed in the device arm. The clinical implication of AF secondary to device closure, including future risk of stroke, is not well known and requires further investigation. At the time of writing, recommendations for PFO closure have not been incorporated into stroke management guidelines.

\section{PFO occlusion devices}

A variety of devices have undergone evaluation for $\mathrm{PFO}$ closure over the past two decades, with self-expanding double-disc PFO occluders representing most of these. These consist of two self-expanding atrial discs which are attached through a central waist, and are positioned by sequential unfolding of the left (distal) and right (proximal) atrial discs after the $\mathrm{PFO}$ is crossed using a guidewire advanced from the right atrium (46-48).

The FDA approved Amplatzer PFO Occluder (St Jude Medical) consists of two Nitinol mesh discs with polyester fabric to promote endothelialisation, and a fixed narrow waist to keep each disc well apposed to the septal wall. The device comes in multiple sizes based on patient anatomy, with the right atrial disc most often larger than the left atrial disc, and is easily recaptured and repositioned for precise 


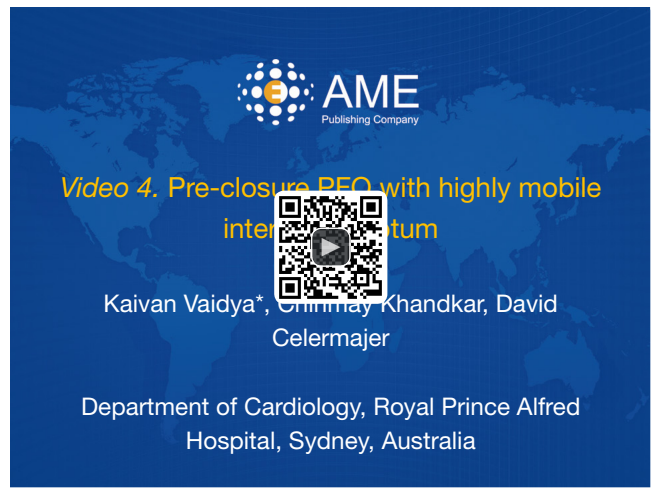

Figure 4 Pre-closure $\mathrm{PFO}$ with highly mobile interatrial septum (49). PFO, patent foramen ovale.

Available online: http://www.asvide.com/article/view/29136

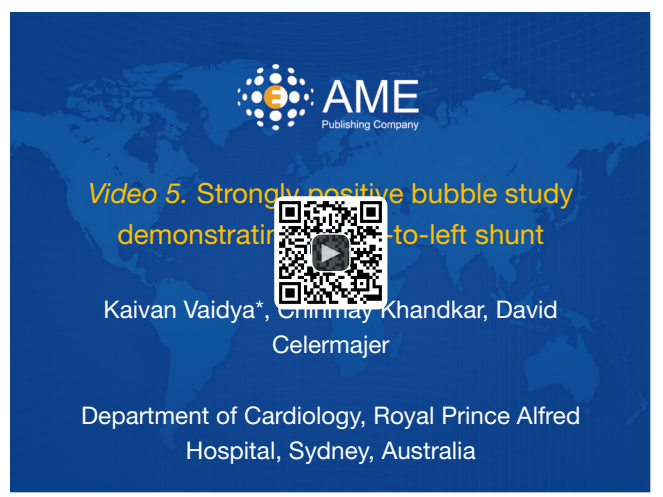

Figure 5 Strongly positive bubble study demonstrating a right-to-left shunt (50).

Available online: http://www.asvide.com/article/view/29137

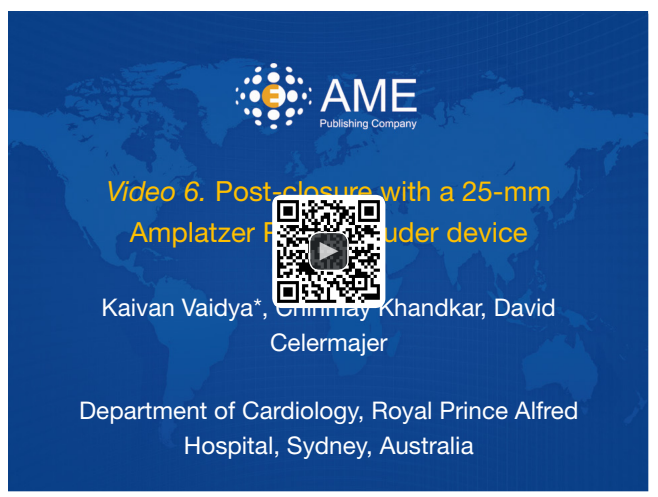

Figure 6 Post-closure with a 25-mm Amplatzer PFO Occluder device (51). PFO, patent foramen ovale.

Available online: http://www.asvide.com/article/view/29138 placement. Figures 4-6 demonstrates a pre-closure, bubble study, and post-closure TOE of a patient who underwent a $25 \mathrm{~mm}$ Amplatzer PFO Occluder implantation. The Cardioform Septal Occluder (Gore \& Associates) is a retrievable double-disc device for transcatheter closure of PFOs up to $17 \mathrm{~mm}$. It consists of two conformable discs in a petal design, made of a nitinol frame covered by a thin expanded polytetrafluoroethylene membrane, which facilitate rapid endothelialisation and reduce friction with adjacent cardiac structures (thereby reducing the risk of erosion). Another double-disc occluders is the Figulla Flex II PFO Occluder (Occlutech AB), which is technically very similar to the Amplatzer device, except for minor design differences including a lower-profile left atrial disc, absent hub on the left atrial side, and hinged attachment to the delivery cable; all of which aim to achieve optimal alignment and reduce risk of device erosion (46-48).

Less commonly used closure devices include the FlatStent occluder (Coherex Medical), which is inserted directly into a relatively long PFO tunnel and stabilised with two adjustable left and right atrial anchors which closely appose the septum primum and secundum (46). The development of a completely or partially bioabsorbable occluder is the focus of ongoing research. With less prosthetic material, these designs aim to minimise thrombus formation, arrhythmias, and erosion, and allow potential future procedural septal punctures as opposed to the double-disc devices.

A 2013 RCT (48) compared three different PFO-closure devices for complete closure, and the composite outcome of stroke, TIA, cerebrovascular death, or any other paradoxical embolism within 5 years of implantation, in 660 patients. There were 220 patients in each group-Amplatzer, CardioSEAL STARFlex, and the Gore Helex Septal Occluder, and PFO closure was performed for cryptogenic stroke $(58 \%)$ and/or TIA (51\%), migraine $(7.6 \%)$, or decompression sickness $(0.5 \%)$. All patients were prescribed aspirin and clopidogrel daily for 6 months post-procedure. Device implantation was technically successful in all interventions. At 5-year follow-up, the highest complete closure rate was achieved with Amplatzer (100\%), and the lowest with Helex occluders (96.8\%). Device embolisation occurred in three patients (all Helex) and thrombus formation on the device was detected in 12 cases (11 STARFlex, 1 Helex, 0 Amplatzer, $\mathrm{P}<0.001$ ). Within 5 years, the primary endpoint occurred less commonly 
$(\mathrm{P}=0.04)$ in the Amplatzer group $(1.4 \%)$ compared to STARFlex $(6 \%)$ and Helex (4\%). The incidence of atrial fibrillation was significantly higher $(\mathrm{P}<0.001)$ in the STARFlex group (12.3\%) than in the Amplatzer (3.6\%) or Helex $(2.3 \%)$ groups. The findings of this study suggested that Amplatzer was the device with most successful implantation and least procedural complications and recurrent neurological event rates.

\section{Medical therapy in cryptogenic stroke}

Patients not suitable for PFO closure are commenced on medical therapy to prevent recurrence of stroke/TIA. Given the hypothesised pathophysiology behind PFOassociated cryptogenic strokes (paradoxical venous emboli), one would anticipate superior efficacy from anticoagulation in comparison to antiplatelet therapy. However, there is mixed evidence in this domain, with no statistically significant evidence strongly favouring either, and hence the preferred antithrombotic strategy for secondary prevention in patients with cryptogenic stroke and PFO remains an unresolved and challenging issue. This question is becoming increasingly pertinent with the advent of direct oral anticoagulants which have effective venous thrombosis prevention, less bleeding risk, and greater convenience of use than warfarin in other clinical situations (23).

The PICCS trial was the first randomized control trial to investigate anticoagulation $v s$. antiplatelet therapy in patients with presumed PFO-mediated cryptogenic stroke (52) and it showed no statistically significant benefit in the use of warfarin $(n=42)$ compared to aspirin $(n=56)$ for reducing two-year rates of recurrent stroke or death (HR 0.52 , 95\% CI: $0.16-1.67, \mathrm{P}=0.28)$, although there was a trend towards event reduction in warfarin-treated patients. Similarly, a small randomised trial of 44 patients (antiplatelet $\mathrm{n}=23$; anticoagulant $=21$ ) with $\mathrm{PFO}$ and cryptogenic stroke compared rate of recurrent stroke/TIA or death between aspirin and warfarin and found no significant difference (HR 0.45, 95\% CI: 0.1-1.8, $\mathrm{P}=0.26$ ) (53). A 2015 meta-analysis pooled data from 12 studies comparing antiplatelet and anticoagulant therapy (33). From the full cohort of 2,385 patients, there was no statistically significant evidence to suggest that anticoagulants $(\mathrm{n}=803)$ were superior to antiplatelet therapy $(\mathrm{n}=1,582)$ for the primary composite outcome (recurrent stroke, TIA, or death) (adjusted HR $0.76,95 \%$ CI: $0.52-1.12$ ) or the secondary outcome of stroke alone (adjusted HR 0.75, 95\% CI: 0.44-1.27). However, this was an intention-to-treat analysis, and anticoagulant use had a statistically significant beneficial effect on the composite outcome in an analysis standardised to the patient population who actually received antiplatelet therapy (adjusted HR 0.64, 95\% CI: 0.42-0.99). Importantly, there was no superiority of anticoagulant over antiplatelet therapy in the higher RoPE score group, who have a lower burden of vascular risk factors and are more likely to have index events due to paradoxical venous emboli, although this may also be confounded by a higher rate of occult atrial fibrillation in the lower RoPE score cohort.

There was no evidence of outcome interaction with antithrombotic treatment (aspirin, warfarin, or both) in the CLOSURE-1 trial (35). In the RESPECT study (42), there was a suggestion of greater stroke risk reduction with the closure group than with the medical therapy group among patients randomly assigned to antiplatelets rather than warfarin. The medical therapy arm of the CLOSE (41) trial had a total of 361 patients (antiplatelet $n=174$; anticoagulant $\mathrm{n}=187$ ) and although there was a signal pointing towards superiority of oral anticoagulation over antiplatelet therapy, however this was not statistically significant (HR 0.37, 95\% CI: 0.07-1.38). Finally, the NAVIGATE-ESUS study was a phase III trial that aimed to evaluate the effectiveness of rivaroxaban against aspirin for prevention of recurrent stroke in patients with cryptogenic stroke in a cohort of 7,213 patients (54). The study was terminated early at eleven months due to a markedly increased bleeding rate observed in the rivaroxaban group (HR 2.72, $95 \%$ CI: $1.68-4.39, \mathrm{P}<0.001)$, but with no difference in the primary efficacy outcome of first recurrence of stroke or systemic embolism (HR 1.07, 95\% CI: 0.87-1.33, $\mathrm{P}=0.52$ ).

\section{Migraine and PFO}

Multiple studies have reported a significant association between migraine headaches, particularly migraine with aura, and the presence of a PFO. A 2016 meta-analysis pooled 21 case-control studies $(n=5,572)$ to show that there is higher prevalence of migraine (OR 2.46, 95\% CI: 1.55 3.91, $\mathrm{P}=0.0001)$ and migraine with aura (OR 3.36, 95\% CI: 2.04-5.55, $\mathrm{P}<0.001)$ in $\mathrm{PFO}$ patients compared to non-PFO patients (55). Studies have also demonstrated a right-to-left shunt in $41-48 \%$ of patients with migraine with aura (56). The prevailing hypothesis regarding the pathophysiological basis of migraines with a $\mathrm{PFO}$ is that the right-to-left shunting in a PFO allows blood with low oxygen content, paradoxical microemboli, and/or venous-system metabolites and chemicals to enter the systemic circulation, thereby irritating cerebral vasculature in susceptible individuals. 
Metabolites such as serotonin, which can increase platelet activation and aggravation and thereby potentially triggering migraine with aura, are usually metabolised by pulmonary monoamine oxidases, however this enzyme may be bypassed by the right-to-left shunt (57-59). As with cryptogenic stroke, there has been considerable interest into whether PFO closure would achieve therapeutic benefit for migraines. After initial promising retrospective observational data suggesting that $80 \%$ of patients reported improvement in migraine attacks after PFO closure (60), there have been three randomised trials to date.

The first of these, the MIST trial (61), recruited patients aged 18 to 60 who suffered from migraine with aura, experienced frequent migraine attacks, had previously failed 2 or more classes of prophylactic treatment, and had a PFO with moderate or large right-to-left shunts. These 147 patients were randomised to either transcatheter closure $(n=74)$ with the STARFlex implant or to a sham procedure ( $n=73)$, with follow-up over 6 months. The primary efficacy endpoint was cessation of migraine headache 90-180 days post-procedure. There was no significant difference in this primary endpoint between the two groups ( 3 in each group, $\mathrm{P}=0.51$ ), or the secondary endpoints (change in severity, frequency, or character of the migraines). Two outliers in the implant group accounted for over one-third of the overall migraines experienced in the follow-up period, and if these were excluded, a significant 2.2 days/month reduction was noted in median total migraine headache days compared with 1.3 days/month in the sham group $(\mathrm{P}=0.027)$. Possible reasons for the overall negative result in MIST include recruitment of patients with severe and refractory migraine which are potentially less amenable to treatment, continued use of prophylactic medications in both arms during the trial, underpowered secondary endpoints due to an unrealistic primary outcome (migraine cessation), and a short period of assessment (90-180 days post-procedure).

Second, the PRIMA trial (62), recruited migraine patients with a $\mathrm{PFO}$ who were unresponsive to preventative medications and randomised 107 to PFO closure $(\mathrm{n}=53)$ with the Amplatzer Occluder or medical therapy $(n=54)$. Both groups were given aspirin for 6 months and clopidogrel for 3 months, and the primary endpoint was reduction in monthly migraine days during months $9-12$ after randomisation compared with a 3 -month baseline phase pre-randomisation. This primary outcome was negative with -2.9 days after closure vs. -1.7 days in the control group $(\mathrm{P}=0.17)$. The average reduction in migraine attacks in closure $(-2.1)$ vs. control $(-1.3)$ arms was also not significant $(\mathrm{P}=0.097)$. However, in the closure arm $38 \%$ experienced a $50 \%$ or greater reduction in number of migraine days relative to baseline compared with $15 \%$ in the control arm $(\mathrm{P}=0.019)$. Post hoc analyses showed a greater mean reduction in migraine with aura days per month $(-2.4 v s .-0.6, \mathrm{P}=0.014)$ and the number of migraine with aura attacks $(-2.0$ vs. $-0.5, \mathrm{P}=0.0003)$ in the closure cohort compared to controls. Despite the overall negative result, these findings of more responders and greater reduction of migraine with aura are pertinent, suggesting that migraines attributable to PFO may be more likely to be preceded by aura. However, the major limitations of the PRIMA trial were the small sample size and the lack of a sham intervention for patient blinding (unlike MIST), and so any hypothesis derived from PRIMA needs to be confirmed in another trial.

Finally, the double-blinded PREMIUM trial (56) recruited 230 patients with a right-to-left shunting PFO who experienced 6 to 14 migraine days per month, and had failed at least three preventative medications. These patients were randomised to medical therapy with a sham procedure $(\mathrm{n}=107)$ or PFO closure with the Amplatzer Occluder ( $\mathrm{n}=123)$ and were followed for 1 year. Primary endpoints were responder rate defined as $50 \%$ reduction in migraine attacks, and secondary endpoints included reduction in migraine days. The primary efficacy outcome was not met: $38.5 \%$ and $32 \%$ of patients in the device and control arms experienced the $50 \%$ reduction in attacks $(\mathrm{P}=0.32)$. However, there was a significant decrease in the mean number of migraine days per month in the device vs. control arm $(-3.4$ vs. $-2.0, \mathrm{P}=0.025)$, and there was a significant difference in subjects who had complete cessation of attacks $(8.5 \%$ vs. $1.0 \%, \mathrm{P}=0.01)$ favouring closure. Only one serious adverse event (transient atrial fibrillation) occurred in the closure group. Approximately two-thirds of patients recruited had migraine with aura, which is higher than in the general population, and likely reflects the fact that these patients are more likely to have a significant right-to-left shunt. A post-hoc subgroup analysis in patients predominantly experiencing migraines with aura $(>50 \%$ of episodes) showed that there was a significant difference in the primary outcome: $49 \%$ vs. $23 \%$ for device $v s$. control groups $(\mathrm{P}=0.015)$. For patients with frequent aura, complete cessation of attacks was seen in $15.4 \%$ of the device cohort vs. $2.5 \%$ in controls $(\mathrm{P}=0.04)$.

The findings from these three trials should be interpreted with caution. Although there is a signal that PFO 
closure may be clinically beneficial in patients suffering predominantly from migraine with aura, future large randomised trials with a sham interventional procedure and appropriately defined prespecified endpoints would be required to investigate this further.

\section{Conclusions}

Patent foramen ovale plays a key role in the pathogenesis of a variety of clinical syndromes, most prominently cryptogenic stroke and migraine headaches, both of which represent a substantial contributor to morbidity, and in the case of stroke, potentially mortality, in susceptible patients. Given the high prevalence of PFO in the general population, and the fact that the potential benefit from transcatheter closure is contingent on several specific clinical or anatomical characteristics, patients suitable for the endovascular procedure should be identified after a comprehensive interdisciplinary assessment by both a neurologist and cardiologist. In the case of ischaemic strokes, there must first be intensive diagnostic exclusion of alternative aetiologies such as small-vessel disease, subclinical arrhythmias, and hypercoagulable states. Secondly, the likelihood that the stroke was attributable to the PFO must be evaluated based on patient age, vascular risk factors and imaging features. Finally, patients with presumably pathogenic PFOs who are likely to benefit most from closure over medical therapy should be identified, and specific features which suggest greatest benefit are the presence of an ASA or a large right-to-left shunt. Most "positive" trials have focused on patients aged 18 to 60 years. Unresolved issues include the clinical utility of antiplatelet $v s$. anticoagulation medical therapy after cryptogenic stroke, the long-term prognostic implications of new-onset atrial fibrillation post device implantation, and whether patients who predominantly experience migraine with aura may benefit from PFO closure. Future randomised clinical trials will ideally help to clarify these issues and reform guidelines accordingly.

\section{Acknowledgements}

None.

\section{Footnote}

Conflicts of Interest: The authors have no conflicts of interest to declare.
Informed Consent: Written informed consent was obtained from the patient for publication of this manuscript and any accompanying images.

\section{References}

1. Hara H, Virmani R, Ladich E, et al. Patent Foramen Ovale: Current Pathology, Pathophysiology, and Clinical Status. J Am Coll Cardiol 2005;46:1768-76.

2. Turner D, Forbes T. Angiographic Evaluation for PFO and Pulmonary AVMs. In: Amin Z, Tobls J, Horst S et al., editors. Patent Foramen Ovale. London: Springer-Verlag, 2015.

3. Rigatelli G. Patent foramen ovale: the evident paradox between the apparently simple treatment and the really complex pathophysiology. J Cardiovasc Med (Hagerstown) 2007;8:300-4.

4. Clark EB. Pathogenetic mechanisms of congenital cardiovascular malformations revisited. Semin Perinatol 1996;20:465-72.

5. Mojadidi MK, Zaman MO, Elgendy IY, et al. Cryptogenic Stroke and Patent Foramen Ovale. J Am Coll Cardiol 2018;71:1035-43.

6. Di Tullio M, Sacco RL, Venketasubramanian N, et al. Comparison of diagnostic techniques for the detection of a patent foramen ovale in stroke patients. Stroke 1993;24:1020-4.

7. Onorato M, Casilli F, Anzola GP. Echocardiographic Detection and Transcranial Doppler Quantification of Right-to-Left Shunting. In: Amin Z, Tobls J, Horst S et al., editors. Patent Foramen Ovale. London: SpringerVerlag, 2015.

8. Lynch JJ, Schuchard GH, Gross CM, et al. Prevalence of right-to-left atrial shunting in a healthy population: detection by Valsalva maneuver contrast echocardiography. Am J Cardiol 1984;53:1478-80.

9. Vaidya K, Khandkar C, Celermajer D. Small-sized patent PFO. Asvide 2018;5:919. Available online: http://www.asvide. com/article/view/29132

10. Vaidya K, Khandkar C, Celermajer D. Moderate-sized PFO. Asvide 2018;5:920. Available online: http://www.asvide.com/ article/view/29134

11. Vaidya K, Khandkar C, Celermajer D. Large-sized PFO. Asvide 2018;5:921. Available online: http://www.asvide. com/article/view/29135

12. Belkin RN, Pollack BD, Ruggiero ML, et al. Comparison of transesophageal and transthoracic echocardiography with contrast and color flow Doppler in the detection of patent foramen ovale. Am Heart J 1994;128:520-5. 
13. Siostrzonek P, Zangeneh M, Gössinger H, et al. Comparison of transesophageal and transthoracic contrast echocardiography for detection of a patent foramen ovale. Am J Cardiol 1991;68:1247-9.

14. Kerr AJ, Buck T, Chia K, et al. Transmitral Doppler: a new transthoracic contrast method for patent foramen ovale detection and quantification. J Am Coll Cardiol 2000;36:1959-66.

15. Saric M, Armour AC, Arnaout MS, et al. Guidelines for the Use of Echocardiography in the Evaluation of a Cardiac Source of Embolism. J Am Soc Echocardiogr 2016;29:1-42.

16. Mojadidi MK, Roberts SC, Winoker JS, et al. Accuracy of transcranial Doppler for the diagnosis of intracardiac right-to-left shunt: a bivariate meta-analysis of prospective studies. JACC Cardiovasc Imaging 2014;7:236-50.

17. Soliman OI, Geleijnse ML, Meijboom FJ, et al. The use of contrast echocardiography for the detection of cardiac shunts. Eur J Echocardiogr 2007;8:S2-12.

18. Gin KG, Huckell VF, Pollick C. Femoral vein delivery of contrast medium enhances transthoracic echocardiographic detection of patent foramen ovale. J Am Coll Cardiol 1993;22:1994-2000.

19. Hamann GF, Schatzer-Klotz D, Frohlig G, et al. Femoral injection of echo contrast medium may increase the sensitivity of testing for a patent foramen ovale. Neurology 1998;50:1423-8.

20. Koh TW. When to use femoral vein injection for diagnosis of patent foramen ovale-Effect of a persistent eustachian valve on right atrial flow patterns during contrast transesophageal echocardiography. Echocardiography 2017;34:768-72.

21. Orford J, Whisenant B. An Overview of Clinical Syndromes (Keeping the Heart in Mind). In: Amin Z, Tobls J, Horst S et al., editors. Patent Foramen Ovale. London: Springer-Verlag, 2015.

22. Sacco RL, Ellenberg JH, Mohr JP, et al. Infarcts of undetermined cause: the NINCDS Stroke Data Bank. Ann Neurol 1989;25:382-90.

23. Lattanzi S, Brigo F, Cagnetti C, et al. Patent Foramen Ovale and Cryptogenic Stroke or Transient Ischemic Attack: To Close or Not to Close? A Systematic Review and Meta-Analysis. Cerebrovasc Dis 2018;45:193-203.

24. Lechat P, Mas JL, Lascault G, et al. Prevalence of patent foramen ovale in patients with stroke. $\mathrm{N}$ Engl J Med 1988;318:1148-52.

25. Lamy C, Giannesini C, Zuber M, et al. Clinical and imaging findings in cryptogenic stroke patients with and without patent foramen ovale: the PFO-ASA Study. Atrial Septal Aneurysm. Stroke 2002;33:706-11.

26. Cramer SC, Rordorf G, Maki JH, et al. Increased pelvic vein thrombi in cryptogenic stroke: results of the Paradoxical Emboli from Large Veins in Ischemic Stroke (PELVIS) study. Stroke 2004;35:46-50.

27. Landzberg MJ, Khairy P. Indications for the closure of patent foramen ovale. Heart 2004;90:219-24.

28. Overell JR, Bone I, Lees KR. Interatrial septal abnormalities and stroke: a meta-analysis of case-control studies. Neurology 2000;55:1172-9.

29. Bhan A, Clapp B. Review of Data and Disussion - Who should Undergo Patent Foramen Ovale Closure in 2014. Interv Cardiol 2014;9:115-20.

30. Kumar T, Budnur SC, Mahadevappa NC, et al. Paradoxical embolism via a patent foramen ovale. BMJ Case Reports $2013 ; 2013$.

31. Sattiraju S, Masri SC, Liao K, et al. Three-dimensional transesophageal echocardiography of a thrombus entrapped by a patent foramen ovale. Ann Thorac Surg 2012;94:e101-2.

32. Kent DM, Ruthazer R, Weimar C, et al. An index to identify stroke-related vs incidental patent foramen ovale in cryptogenic stroke. Neurology 2013;81:619-25.

33. Kent DM, Dahabreh IJ, Ruthazer R, et al. Anticoagulant vs. antiplatelet therapy in patients with cryptogenic stroke and patent foramen ovale: an individual participant data meta-analysis. Eur Heart J 2015;36:2381-9.

34. Thaler DE, Ruthazer R, Weimar C, et al. Recurrent stroke predictors differ in medically treated patients with pathogenic vs other PFOs. Neurology 2014;83:221-6.

35. Furlan AJ, Reisman M, Massaro J, et al. Closure or Medical Therapy for Cryptogenic Stroke with Patent Foramen Ovale. N Engl J Med 2012;366:991-9.

36. Meier B, Kalesan B, Mattle HP, et al. Percutaneous Closure of Patent Foramen Ovale in Cryptogenic Embolism. N Engl J Med 2013;368:1083-91.

37. Carroll JD, Saver JL, Thaler DE, et al. Closure of Patent Foramen Ovale vs. Medical Therapy after Cryptogenic Stroke. N Engl J Med 2013;368:1092-100.

38. Khan AR, Bin Abdulhak AA, Sheikh MA, et al. Device Closure of Patent Foramen Ovale Versus Medical Therapy in Cryptogenic Stroke: A Systematic Review and Meta-Analysis. JACC: Cardiovascular Interventions 2013;6:1316-23.

39. Stortecky S, da Costa BR, Mattle HP, et al. Percutaneous closure of patent foramen ovale in patients with cryptogenic embolism: a network meta-analysis. Eur Heart 
J 2015;36:120-8.

40. Kent DM, Dahabreh IJ, Ruthazer R, et al. Device Closure of Patent Foramen Ovale After Stroke: Pooled Analysis of Completed Randomized Trials. J Am Coll Cardiol 2016;67:907-17.

41. Mas JL, Derumeaux G, Guillon B, et al. Patent Foramen Ovale Closure or Anticoagulation vs. Antiplatelets after Stroke. N Engl J Med 2017;377:1011-21.

42. Saver JL, Carroll JD, Thaler DE, et al. Long-Term Outcomes of Patent Foramen Ovale Closure or Medical Therapy after Stroke. N Engl J Med 2017;377:1022-32.

43. Søndergaard L, Kasner SE, Rhodes JF, et al. Patent Foramen Ovale Closure or Antiplatelet Therapy for Cryptogenic Stroke. N Engl J Med 2017;377:1033-42.

44. Turc G, Calvet D, Guerin P, et al. Closure, Anticoagulation, or Antiplatelet Therapy for Cryptogenic Stroke With Patent Foramen Ovale: Systematic Review of Randomized Trials, Sequential Meta-Analysis, and New Insights From the CLOSE Study. J Am Heart Assoc 2018;7.

45. Ahmad Y, Howard JP, Arnold A, et al. Patent foramen ovale closure vs. medical therapy for cryptogenic stroke: a meta-analysis of randomized controlled trials. Eur Heart J 2018;39:1638-49.

46. Melikian NM, Philip. The Spectrum of PFO Closure Devices. 2017. Available online: https://citoday. com/2017/06/the-spectrum-of-pfo-closure-devices/

47. Taaffe M, Fischer E, Baranowski A, et al. Comparison of Three Patent Foramen Ovale Closure Devices in a Randomized Trial (Amplatzer Versus CardioSEALSTARflex Versus Helex Occluder). Am J Cardiol 2008;101:1353-8.

48. Hornung M, Bertog SC, Franke J, et al. Long-term results of a randomized trial comparing three different devices for percutaneous closure of a patent foramen ovale. Eur Heart J 2013;34:3362-9.

49. Vaidya K, Khandkar C, Celermajer D. Pre-closure PFO with highly mobile interatrial septum. Asvide 2018;5:922. Available online: http://www.asvide.com/article/ view/29136

50. Vaidya K, Khandkar C, Celermajer D. Strongly positive bubble study demonstrating a right-to-left shunt. Asvide 2018;5:923. Available online: http://www.asvide.com/

Cite this article as: Vaidya K, Khandkar C, Celermajer D. Current management aspects in adult congenital heart disease: non-surgical closure of patent foramen ovale. Cardiovasc Diagn Ther 2018;8(6):739-753. doi: 10.21037/cdt.2018.09.09 article/view/29137

51. Vaidya K, Khandkar C, Celermajer D. Post-closure with a $25-\mathrm{mm}$ Amplatzer PFO Occluder device. Asvide 2018;5:924. Available online: http://www.asvide.com/ article/view/29138

52. Homma S, Sacco RL, Di Tullio MR, et al. Effect of medical treatment in stroke patients with patent foramen ovale: patent foramen ovale in Cryptogenic Stroke Study. Circulation 2002;105:2625-31.

53. Shariat A, Yaghoubi E, Farazdaghi M, et al. Comparison of medical treatments in cryptogenic stroke patients with patent foramen ovale: A randomized clinical trial. J Res Med Sci 2013;18:94-8.

54. Hart RG, Sharma M, Mundl H, et al. Rivaroxaban for Stroke Prevention after Embolic Stroke of Undetermined Source. N Engl J Med 2018;378:2191-201.

55. Takagi H, Umemoto T. A meta-analysis of case-control studies of the association of migraine and patent foramen ovale. J Cardiol 2016;67:493-503.

56. Tobis JM, Charles A, Silberstein SD, et al. Percutaneous Closure of Patent Foramen Ovale in Patients With Migraine. J Am Coll Cardiol 2017;70:2766.

57. Sharma A, Gheewala N, Silver P. Role of patent foramen ovale in migraine etiology and treatment: a review. Echocardiography 2011;28:913-7.

58. Peter W, Simon N. The Role of Cardiac and Pulmonary Pathology in Migraine: A Hypothesis. Headache 2006;46:429-34.

59. Mojadidi MK, Dave N, Gevorgyan R, et al. The Association of Patent Foramen Ovale and Migraine Headache. In: Amin Z, Tobls J, Horst S et al., editors. Patent Foramen Ovale. London: Springer-Verlag, 2015.

60. Shi Lv J, Han et al. Migraine and percutaneous patent foramen ovale closure: a systematic review and metaanalysis. BMC Cardiovascular Disorders 2017;17:203.

61. Dowson A, Mullen MJ, Peatfield R, et al. Migraine Intervention With STARFlex Technology (MIST) Trial. Circulation 2008;117:1397.

62. Mattle HP, Evers S, Hildick-Smith D, et al. Percutaneous closure of patent foramen ovale in migraine with aura, a randomized controlled trial. Eur Heart J 2016;37:2029-36. 